Antonio Sergio Almeida Fonseca ${ }^{\mathrm{a}}$

Danilo Fernandes Costa ${ }^{b}$

Virgínia Dapper ${ }^{c}$

Jorge Mesquita Huet Machado ${ }^{d}$

Daniel Valente

Leandro Vargas Barreto de Carvalho a

Isabele Campos Costa-Amaral ${ }^{\text {a }}$

Sergio Rabello Alves ${ }^{\text {a }}$

Paula de Novaes Sarcinelli ${ }^{\text {a }}$

Marco Antônio Carneiro Menezes a

Ariane Leites Larentis ${ }^{\text {a }}$

Maria Juliana Moura-Correa e,

Rita de Cássia Oliveira da Costa Mattos ${ }^{a}$

a Fundação Oswaldo Cruz (Fiocruz). Escola Nacional de Saúde Pública Sergio Arouca (ENSP). Centro de Estudos da Saúde do Trabalhador e Ecologia Humana (CESTEH). Rio de Janeiro, RJ, Brasil.

${ }^{\mathrm{b}}$ Ministério do Trabalho. Superintendência Regional do Trabalho e Emprego de SP. Seção de Segurança e Saúde do Trabalhador (Segur). São Paulo, SP, Brasil.

' Secretaria Estadual da Saúde do Rio Grande do Sul. Centro Estadual de Vigilância em Saúde. Divisão de Vigilância em Saúde do Trabalhador. Porto Alegre, RS, Brasil.

${ }^{d}$ Fundação Oswaldo Cruz (Fiocruz).

Diretoria Regional de Brasília. Brasília, DF, Brasil.

e Universidade Federal da Bahia (UFBA). Instituto de Saúde Coletiva (ISC). Programa Integrado em Saúde Ambiental e do Trabalhador (PISAT). Salvador, BA, Brasil.

f Secretaria Municipal de Saúde. Porto Alegre, RS, Brasil.

Contato: Ariane Leites Larentis

E-mail: arilarentis@yahoo.com.br

Os autores do artigo declaram que não há conflito de interesses.

Este estudo recebeu fomento através de cooperação interinstitucional (2010-2012) com a Coordenação Geral de Vigilância em Saúde Ambiental (CGVAM/SVS/Ministério da Saúde), do Programa Inova-ENSP (Edital 2013-2015 do Programa de Apoio à Pesquisa, Desenvolvimento e Inovação em Saúde Pública da ENSP/Fiocruz), Edital FAPERJ N 32/2013- Programa Apoio às Instituições de Ensino e Pesquisa Sediadas no Estado do Rio de Janeiro - 2013 (Processo E-26/11.732/2013) e do Chamamento Público nº 05/2014 Iniciativas Educacionais Aplicadas à Vigilância em Saúde da Secretaria de Vigilância em Saúde do Ministério da Saúde (SVS/MS), por meio do Centro de Estudos da Saúde do Trabalhador e Ecologia Humana (CESTEH/ ENSP/Fiocruz), Centro Colaborador da OPAS/ OMS para Saúde Pública e Ambiental.

Os autores informam que o trabalho não foi baseado em tese e não foi apresentado em reunião científica.

Recebido: 06/12/2015

Revisado: 26/09/2016

Aprovado: 27/09/2016

\section{Classificação clínico-laboratorial para manejo clínico de trabalhadores expostos ao benzeno em postos de revenda de combustíveis}

\author{
Clinical-laboratory classification for clinical management of \\ workers exposed to benzene in gas stations
}

\section{Resumo}

Introdução: trabalhadores de postos de revenda de combustíveis estão expostos a compostos orgânicos voláteis, dos quais se destaca o benzeno. O manejo clínico do amplo espectro de sinais e sintomas desses trabalhadores ainda é um desafio para assistência em saúde. Objetivo: apresentar proposta de classificação clínico-laboratorial baseada na análise de hemograma e na avaliação clínica dos trabalhadores, para orientar a conduta dos profissionais de saúde. Métodos: a classificação clínico-laboratorial foi definida a partir de critérios de hierarquização dos resultados das avaliações clínicas e de vigilância à saúde de modo a possibilitar a organização dos procedimentos e fluxos de atenção à saúde. Resultados: a proposta define o manejo clínico com base na classificação dos expostos ao benzeno em quatro níveis de alterações clínico-laboratoriais que determinam os procedimentos compatíveis com o grau da exposição e de seus efeitos à saúde: 1) sem alterações significativas; 2) alterações inespecíficas ou incompletas, 3) alterações clínicas e/ou laboratoriais; 4) alterações sugestivas de benzenismo. Conclusão: a proposta de classificação dos achados clínico-laboratoriais é potencialmente capaz de orientar a conduta médica na avaliação das condições de saúde e dos riscos da exposição ao benzeno e de subsidiar o monitoramento sistemático e contínuo necessário para a definição de rotinas assistenciais para os coletivos de trabalhadores.

Palavras-chave: manejo clínico; hemograma; benzenismo; saúde do trabalhador.

\begin{abstract}
Introduction: gas stations workers are exposed to volatile organic compounds, especially benzene. Clinical management of the broad spectrum of symptoms and signs observed in these workers is still a challenge for health care. Objective: to present a clinical-laboratory classification, based on hemogram analysis and clinical evaluation of workers exposed to benzene, as guidelines for health care professionals. Methods: the clinical-laboratory classification was defined based on priority-setting criteria for the results of clinical evaluations and health surveillance in order to allow the organization of health care flows and procedures. Results: the proposal defines the clinical management based on classification of workers exposed to benzene in four levels of clinical and laboratory alterations that determine procedures according to level of exposure and health effects: 1) without significant alterations; 2) unspecific or incomplete alterations; 3) clinical and/or laboratory alterations; and 4) alterations suggesting benzene poisoning. Conclusion: the proposal for classification of clinical and laboratory findings is potentially able to guide medical practice in the assessment of health conditions and risks of benzene exposure, and subsidize the systematic and continuous monitoring necessary for establishing health care practices offered to workers.
\end{abstract}

Keywords: clinical management; hemogram; benzene poisoning; worker's health. 


\section{Introdução}

A gasolina é um derivado do petróleo que contém hidrocarbonetos, incluindo o benzeno, tolueno, etilbenzeno e xilenos (BTEX), e outros compostos orgânicos voláteis (COVs). A exposição simultânea a essa mistura parece ter um impacto significativo na sua toxicidade ${ }^{1}$. O benzeno é reconhecido como um carcinógeno pertencente ao Grupo 1 pela International Agency for Research on Cancer (IARC), presente no ambiente e largamente utilizado na indústria, na fabricação de produtos químicos e na composição do petróleo bruto e da gasolina ${ }^{2,3}$.

Contaminações ambientais e ocupacionais relacionadas aos COVs em postos de combustíveis continuam sendo de grande preocupação para as áreas da saúde pública e da saúde do trabalhador, especialmente as contaminações relacionadas ao benzeno presente na gasolina. No Brasil, de acordo com Moura-Correa et al. ${ }^{4}$, a exposição ocupacional relacionada a postos de combustíveis atinge 184.733 frentistas, distribuídos em 39.450 postos de revenda de combustíveis (PRC) em todo o país. Os trabalhadores dos PRC realizam atividades de alto risco por estarem em contato direto com os vapores de BTEX e outros COVs, nas atividades de abastecimento, medição de tanque, transferência de produto, entre outras. Um automóvel, ao abastecer, troca a gasolina líquida pelo vapor saturado que existe dentro do tanque, que pode variar de poucos litros a dezenas de litros, dependendo do volume de gasolina adquirido. Por sua vez, um caminhão-tanque, durante o descarregamento de combustível, pode emanar para a atmosfera grande quantidade de vapor saturado de gasolina, devido sua capacidade de conter dezenas de milhares de litros. Segundo Correa et al. ${ }^{5}$, a mensuração de emissões de vapores de BTEX em postos do Rio de Janeiro identificou valores médios em torno de $30 \mu \mathrm{g} \cdot \mathrm{m}^{-3}$ de benzeno, $48 \mu \mathrm{g} \cdot \mathrm{m}^{-3}$ de tolueno, $23 \mu \mathrm{g} \cdot \mathrm{m}^{-3}$ de etilbenzeno, $47 \mu \mathrm{g} \cdot \mathrm{m}^{-3}$ para $\mathrm{m}$, $p$-xilenos e $14 \mu \mathrm{g} \cdot \mathrm{m}^{-3}$ de $o$-xileno. Costa $^{6}$ evidenciou que, durante o abastecimento de gasolina, os frentistas ficavam expostos a concentrações de benzeno no ar que variavam de 40 a 700 ppb na zona respiratória. Essas atividades e situações de risco levam a uma exposição ubíqua ao benzeno, presente nos processos produtivos e no ambiente. Desse modo, por ser um carcinógeno, não há concentração limite segura que elimine o risco de exposição e de adoecimento dos trabalhadores de PRC e da população no entorno.

Estratégias de redução dos solventes presentes na gasolina, especialmente do nível de exposição ao benzeno, têm sido realizadas com o objetivo de assegurar uma melhoria na qualidade de vida desses trabalhadores, assim como a melhoria da tecnologia dos meios de produção. Essas mudanças são resultado da pressão das políticas de vigilância à saúde ocupacional e da tendência mundial por substituição do benzeno nos processos industriais ${ }^{7}$.

Apesar dos esforços para regulação das emissões de benzeno, um vasto número de trabalhadores continua sendo exposto, em diversas situações precárias de trabalho, incluindo os trabalhadores de postos de combustíveis ${ }^{8}$. As diferentes exposições ao benzeno ainda não são tratadas de maneira sistematizada pelas autoridades e a situação é ainda mais preocupante quando essa exposição está relacionada a baixas concentrações por um longo período de tempo, como no caso da exposição crônica ${ }^{9}$, situação na qual são observados sérios agravos à saúde humana com vários tipos de lesões no tecido da medula óssea que, consequentemente, acarretam as subsequentes alterações no sistema hematopoiético.

A exposição ocupacional pode ocorrer através da inalação e/ou contato dérmico em locais de trabalho onde o benzeno é produzido ou utilizado. Dados de monitoramento indicam que a população em geral pode estar exposta ao benzeno através da inalação de ar, ingestão de alimentos, de água e pelo contato dérmico com produtos de consumo que contenham benzeno ${ }^{10}$. A absorção sistêmica do benzeno está associada a efeitos tóxicos agudos e alterações crônico-degenerativas do sistema hematopoiético, como anemia aplásica, síndrome mielodisplásica, linfoma não Hodgkin e leucemia mieloide aguda. Todas ocasionadas por produtos da biotransformação do benzeno $^{3,11,12}$. Além de ser reconhecido por seus efeitos mielotóxico, leucemogênico e cancerígeno, mesmo em baixas concentrações, o benzeno também está associado a um conjunto de sinais, sintomas e complicações ${ }^{13}$, embora estes nem sempre estejam presentes ${ }^{14}$.

No Brasil, a síndrome clínica da intoxicação por benzeno foi também denominada de benzenismo, numa concepção que envolve suas dimensões bio-sócio-ambientais ${ }^{14-16}$. A complexidade inserida pelas dimensões dessa definição é resultado da ação protagonista de trabalhadores e técnicos, em sindicatos e serviços públicos locais e regionais, na disputa pelo reconhecimento da "leucopenia" como expressão do adoecimento dos trabalhadores intoxicados por benzeno na década de 1980.

Esse movimento foi equivalente à épica luta dos mineiros de carvão pelo reconhecimento das doenças pulmonares específicas, negadas durante décadas por médicos, instituições de ensino e serviços ingleses $^{17}$. Além de constituir uma das principais origens epistêmicas da saúde do trabalhador no Brasil, que repercute até hoje, a experiência em Cubatão permitiu ao longo do tempo uma ampliação do conhecimento sobre a complexidade desses processos de intoxicação ${ }^{18}$. Não obstante tal ampliação, as alterações hematológicas e a leucopenia, em especial, continuam a 
ser referências para os trabalhadores no objetivo de revelar a problemática dessa exposição ${ }^{14}$.

O quadro clínico de toxicidade da exposição ao benzeno se caracteriza por uma repercussão orgânica múltipla, na qual o comprometimento da medula óssea é o componente mais frequente e significativo, sendo a causa básica de diversas alterações hematológicas. A amplitude dos danos provocados pela contaminação por benzeno vem se comprovando de forma reiterada, assim como as evidências de formas graves de adoecimento com exposições a concentrações muito baixas ${ }^{19-21}$.

No Brasil, estudos com trabalhadores expostos ao benzeno já apontavam a presença de astenia, mialgia, sonolência, tontura e infecções de repetição como os sinais e sintomas mais frequentes relacionados à exposição ao benzeno ${ }^{22,23}$. Em relação aos dados laboratoriais hematológicos mais relevantes, foi relatado o aparecimento de leucopenia e demais citopenias, macrocitose, pontilhado basófilo, pseudo Pelger e plaquetopenia. A leucopenia com neutropenia e, em menor frequência, a plaquetopenia isolada ou associada à neutropenia, correspondem à principal repercussão hematológica da hipoplasia secundária ao benzeno ${ }^{14}$. Dessa forma, o hemograma, preferencialmente em série histórica, é o principal instrumento de avaliação para classificação clínico-laboratorial.

Com relação às alterações neuropsicológicas e neurológicas, são observadas alterações de atenção, memória, cefaleia, depressão, insônia, agitação, alterações de comportamento e quadros de polineuropatias periféricas e mielites transversas. Podem ocorrer alterações dermatológicas, como eritema e dermatite irritativa de contato por exposições ocupacionais repetidas e prolongadas ao benzeno ${ }^{14,24}$. No sistema auditivo podem aparecer alterações periféricas ou centrais; podem ser observadas perdas auditivas neurossensoriais, zumbidos, vertigens e dificuldades no processamento auditivo, principalmente na exposição a outros COVs presentes na gasolina, como o tolueno ${ }^{25,26}$.

A compreensão de que o benzenismo é um agravo diretamente relacionado ao processo de trabalho faz com que a definição da abordagem deste problema se oriente por referências que consideram a integralidade da investigação em saúde e a complexidade do processo saúde-doença.

Diferentes classificações de risco têm sido utilizadas, em sua maioria, para triagem separando os casos graves ou urgentes daqueles menos urgentes para definir o atendimento prioritário e os tratamentos mais adequados, de acordo com as especificidades dos grupos. Uma classificação de risco para exposição ao benzeno foi elaborada por Wiwanitkit ${ }^{27}$, a partir dos riscos relativos padronizados, oriundos de estudos publicados anteriormente, que quantificavam a exposição usando o nível do ácido trans, trans-mucônico urinário (AttM). O estudo classificou as ocupações segundo baixo risco (menor que a mediana) e alto risco (igual ou superior à mediana), tendo os frentistas sido classificados como alto risco, pois estão entre as ocupações nas quais o trabalhador tem contato direto com benzeno no ar/ambiente, e cuja fonte é o combustível derivado de petróleo ${ }^{27}$.

A escolha de um biomarcador específico para o benzeno tem sido discutida no Brasil ${ }^{28}$, e tanto o AttM quanto outros indicadores propostos, como o ácido $S$-fenilmercaptúrico, não são facilmente mensuráveis para aplicação em larga escala nos serviços de saúde no país, devido à necessidade de técnicas analíticas específicas. Já o hemograma é um exame de fácil execução, em termos de vigilância, o que permite a proposta de classificação centrar-se nesse procedimento, apesar de ser um indicador de efeito. Entretanto, ainda não foi encontrada em revisão da literatura referência à classificação de risco para orientar o manejo clínico de trabalhadores expostos ao benzeno baseada apenas em hemograma e para uso amplo nas rotinas dos serviços de saúde. No entanto, subsídios de estudos sobre avaliação da exposição ao benzeno através de dados hematimétricos, bioquímicos e citogenéticos fortalecem a perspectiva de utilização destes enquanto procedimentos para manejo clínico dos trabalhadores expostos ${ }^{29}$.

A complexidade dessa exposição e a necessidade de reunir um conjunto de dados clínicos e de exames para análise dos resultados levou à construção de uma proposta de manejo clínico dos trabalhadores expostos ao benzeno e demais COVs, tendo como base o protocolo de atenção à saúde dos trabalhadores expostos ao benzeno ${ }^{14}$. Essa classificação parte da avaliação clínica de forma integral e da sistematização dos resultados dos exames laboratoriais hematológicos. Destaca-se, nesse contexto, a importância fundamental da avaliação integrada, componente da Vigilância em Saúde do Trabalhador, para a identificação de agravos relacionados ao trabalho, níveis de exposição e o desenvolvimento de medidas preventivas conforme grau de risco. Importante salientar que essa proposta de manejo clínico deve ser considerada enquanto processo síntese das experiências recentes, as quais podem e devem ser aperfeiçoadas à medida que novos indicadores sejam desenvolvidos e comprovados para detectar exposição ao benzeno.

Diante do exposto, o objetivo deste ensaio é apresentar uma proposta de classificação clínico-laboratorial para orientar os serviços da Rede Nacional de Saúde do Trabalhador (RENAST) quanto ao manejo clínico de trabalhadores expostos ao benzeno e outros COVs em Postos de Revenda de Combustíveis (PRC), a partir da integralidade das avaliações e dos dados clínicos e hematológicos, bem como do resgate da historicidade destes no processo de exposição nos ambientes de trabalho. 


\section{Métodos}

Adotou-se, para fins dessa classificação, a definição de manejo clínico como um conjunto de medidas que orienta a tomada de decisões na consulta clínica, na organização dos serviços assistenciais e as referências de especialistas em saúde, bem como a vigilância epidemiológica e de processo de trabalho, com a finalidade de prestação da assistência, visando reduzir a morbimortalidade causada por um agravo. Para sua construção, considerou-se a necessidade de organizar rotinas e fluxos de conduta em saúde, baseadas no protocolo de atenção à saúde dos trabalhadores expostos ao benzeno ${ }^{14}$ e no protocolo de vigilância em saúde ${ }^{30}$.

Este manejo clínico está baseado em uma classificação clínico-laboratorial que usou como referência não somente a análise de hemograma, eleita por sua facilidade de execução e ampla utilização de rotina na Vigilância em Saúde do Trabalhador que permite abranger o monitoramento de um grande contingente de trabalhadores, mas também a avaliação clínica. Esta última contempla queixas em geral relacionadas ao benzeno e outros COVs, revisão de sistemas, história médica atual e pregressa, histórico familiar e hábitos de vida, histórico ocupacional e exame físico.

Para definição da classificação clínico-laboratorial, adotou-se como critério hematimétrico a contagem total de leucócitos na faixa de $4.500-11.000 / \mathrm{mm}^{3}$, de acordo com o protocolo, considerando como leucopenia os valores abaixo do limite mínimo ${ }^{14}$. A adoção do critério mais elevado do limite inferior visa a aumentar a sensibilidade da avaliação para garantir a inclusão de possíveis falsos negativos na população investigada.

A Classificação foi organizada em quatro níveis:

Grupo 1 - Sem alterações clínico-laboratoriais significativas;

Grupo 2 - Alterações clínico-laboratoriais inespecíficas ou avaliações incompletas;

Grupo 3 - Alterações clínicas com/sem alterações laboratoriais; ou alterações laboratoriais sem alterações clínicas;

Grupo 4 - Alterações clínico-laboratoriais sugestivas de benzenismo.

O Grupo 4 inclui aqueles trabalhadores com queda relevante e persistente da leucometria, constatada por meio de três exames com intervalo de 15 dias, com ou sem outras alterações associadas. Este critério de avaliação é baseado na recomendação da Portaria 776/2004 ${ }^{31}$ do Ministério da Saúde. Um índice arbitrário de $20 \%$ de redução da leucometria deve ser usado para considerar queda significativa, em relação aos critérios anteriores. Variações menores e a presença de outras alterações hematológicas devem ser consideradas, quando houver suspeita de relevância. Na análise de séries históricas consolidadas com grandes períodos de acompanhamento, deve ser considerado o patamar de pré-exposição ou o mais próximo possível desse período. Devem ser observadas as mudanças tecnológicas nas técnicas dos exames e a hora das coletas. A investigação de intoxicação ocupacional pelo benzeno/benzenismo deve ser realizada mediante a presença de alterações hematológicas em hemogramas seriados, mesmo na ausência de outros achados clínicos que as justifiquem, como:

- Aumento do volume corpuscular médio (macrocitose);

- Diminuição do número absoluto de linfócitos (linfopenia ou linfocitopenia);

- Leucocitose persistente;

- Alterações neutrofílicas: pontilhado basófilo, hiposegmentação dos neutrófilos (pseudo Pelger);

- Presença de macroplaquetas e/ou de plaquetopenia;

- Leucopenia com ou sem associação de outras citopenias.

Importante salientarmos que, de acordo com o protocolo de atenção à saúde dos trabalhadores expostos ao benzeno ${ }^{14}$, as patologias ou as situações clínicas e fisiológicas que concorrem com leucopenia como colagenoses, viroses, alcoolismo, exposição a medicamentos e a outros agentes mielotóxicos, assim como as neutropenias constitucionais e as demais situações anteriormente descritas, devem ser objeto de diagnóstico diferencial e análise sistemática sem, contudo, permitir que sua comprovação seja suficiente para afastar a hipótese de associação com a toxicidade benzênica.

Os sintomas clínicos valorizados são a presença de cefaleia, tonturas, alterações de memória, zumbido, mialgia, fraqueza, sonolência e infecções de repetição, sinais de hipoacusia e a presença de dermatose de contato que, embora não específicos, frequentemente estão associados à intoxicação crônica por benzeno, segundo a Portaria 776/2004 ${ }^{31}$ do Ministério da Saúde. Esses sintomas e sinais devem ser valorizados não só por conta da associação única ao benzeno, mas também por suas repercussões à exposição aos combustíveis.

\section{Resultados}

A classificação dos trabalhadores expostos em cada grupo de acordo com avaliação clínico-laboratorial está sistematizada no Quadro 1. 
Quadro 1 Classificação de trabalhadores expostos ao benzeno de acordo com avaliação clínico-laboratorial

\begin{tabular}{|c|l|l|l|l|l|}
\hline Grupos & \multicolumn{1}{|c|}{ Classificação } & \multicolumn{1}{|c|}{ Sinal e sintoma } & Alteração hematológica & \multicolumn{1}{c|}{ Hemograma } & Periodicidade do hemograma \\
\hline 1 & $\begin{array}{l}\text { Sem alterações } \\
\text { significativas } \\
\text { clínico-laboratoriais }\end{array}$ & Assintomático & Ausente & Ausente & A cada 6 meses \\
\hline $\begin{array}{l}\text { Alterações } \\
\text { clínico-laboratoriais } \\
\text { inespecíficas } \\
\text { ou avaliações } \\
\text { incompletas }\end{array}$ & $\begin{array}{l}\text { Queixas clínicas outras } \\
\text { não relacionadas nas } \\
\text { descrições dos grupos } \\
\text { 3e } 4\end{array}$ & $\begin{array}{l}\text { Não realização dos exames } \\
\text { preconizados }\end{array}$ & $\begin{array}{l}\text { Resultado de } \\
\text { apenas um } \\
\text { hemograma }\end{array}$ & $\begin{array}{l}\text { Intervalo menor de } \\
6 \text { meses dependendo } \\
\text { da avaliação }\end{array}$ \\
\hline $\begin{array}{l}\text { Alterações clínicas } \\
\text { laboratoriais } \\
\text { e alterações } \\
\text { laboratoriais sem } \\
\text { alterações clínicas }\end{array}$ & $\begin{array}{l}\text { Cefaleia, tonturas, } \\
\text { alterações de memória, } \\
\text { zumbido, astenia, } \\
\text { mialgia, fraqueza, } \\
\text { sonolência e infecções } \\
\text { de repetição }\end{array}$ & $\begin{array}{l}\text { Neutropenia, leucopenia, } \\
\text { eosinofilia, linfocitopenia, } \\
\text { monocitopenia, } \\
\text { macrocitose pontilhado } \\
\text { basófilo, pseudo Pelger, } \\
\text { plaquitopenia }\end{array}$ & $\begin{array}{l}\text { Leucócitos } \\
\text { superior ou igual } \\
\text { a 4.500/mm e/ou } \\
\text { Neutrófilos abaixo } \\
\text { de 1.000/mm³ }\end{array}$ & $\begin{array}{l}\text { Intervalo máximo } \\
\text { de 6 meses }\end{array}$ \\
\hline 4 & $\begin{array}{l}\text { Alterações } \\
\text { clínico-laboratoriais } \\
\text { sugestivas de } \\
\text { benzenismo }\end{array}$ & $\begin{array}{l}\text { Cefaleia, tonturas, } \\
\text { alterações de } \\
\text { memória, zumbido, } \\
\text { asteniamialgia, } \\
\text { fraqueza, sonolência e } \\
\text { infecções de repetição }\end{array}$ & $\begin{array}{l}\text { Neutropenia, leucopenia, } \\
\text { eosinofilia, linfocitopenia, } \\
\text { monocitopenia, } \\
\text { macrocitose, pontilhado } \\
\text { basófilo, pseudo Pelger, } \\
\text { plaquitopenia }\end{array}$ & $\begin{array}{l}\text { Leucometria com } \\
\text { redução de 20\%. } \\
\text { Série histórica, } \\
\text { com pelo menos } \\
\text { três exames em } \\
\text { intervalo de 15 dias }\end{array}$ & $\begin{array}{l}\text { Monitoramento contínuo } \\
\text { com série histórica ou } \\
\text { três hemogramas com } \\
\text { intervalo de 15 dias }\end{array}$ \\
\hline
\end{tabular}

Grupo 1: sem alterações significativas clínico-laboratoriais

Conduta clínica: esses trabalhadores deverão ser acompanhados periodicamente.

Periodicidade de monitoramento: realizar hemograma a cada seis meses.

Grupo 2: Alterações clínico-laboratoriais inespecíficas ou avaliações incompletas

Conduta clínica: solicitar imediatamente nova coleta laboratorial ou marcar avaliação clínica quando não realizada e verificar a persistência ou desaparecimento da alteração. Caso a alteração regrida ou o exame clínico for normal, proceder como Grupo 1. Caso a alteração permaneça ou surjam novas alterações clínicas ou laboratoriais, classificar como Grupo 3 ou Grupo 4, de acordo com o tipo de alteração existente.

Periodicidade de Monitoramento: de acordo com os resultados da reavaliação.

Observação: são diversas as alterações clínico-laboratoriais inespecíficas e potenciais. Entre as mais comuns destacam-se as alterações hepáticas, renais, do Sistema Nervoso Central, neurocomportamentais, pulmonares e dermatológicas. Também importante alertar que a exposição a benzeno em PRCs está sempre associada à exposição a múltiplos solventes, o que deve ser levado em consideração na investigação clínica.
Grupo 3: Alterações clínicas com/sem alterações laboratoriais; ou alterações laboratoriais sem alterações clínicas

Conduta clínica: avaliação clínica e verificação da possibilidade de existência de comorbidades e da necessidade de novos exames complementares e pareceres de outras especialidades. Analisar a presença de relação com trabalho e/ou ambiente.

Periodicidade de monitoramento: agendar atendimento ambulatorial de acordo com a gravidade, com intervalo máximo de seis meses.

Observação: deve-se sempre ter presente que a exposição desse grupo ocupacional é decorrente das misturas de solventes. Então, o achado de alterações clínicas comuns a esses produtos ou a alterações típicas de algumas substâncias desse grupo químico reforça a suspeita de que tenha ocorrido exposição e contaminação dos trabalhadores por solventes (eventos sentinela).

\section{Grupo 4: alterações clínico-laboratoriais sugestivas de benzenismo}

Conduta clínica: prioridade absoluta para o atendimento. Avaliar clinicamente e identificar a relação com o trabalho. Caso confirmado, notificar e afastar o trabalhador imediatamente do trabalho. Solicitar rotina de exames complementares, conforme orientação do protocolo de atenção à saúde dos trabalhadores expostos ao benzeno ${ }^{14}$. 
Exames solicitados: hemograma com análise quantitativa e qualitativa das três séries sanguíneas e contagem de reticulócitos (na ausência da série histórica, realizar três hemogramas com intervalo de quinze dias); transaminases (AST e ALT), gama glutamil transferase, bilirrubinas totais e frações e LDH; provas de atividade reumática ou inflamatória: VHS, proteína C reativa e FAN; marcadores de hepatite B e C (anti-HBS Ag, anti-HBc-IgM e anti-HCV); sorologia anti-HIV.

Avaliações solicitadas: neurológica e audiológica.

Periodicidade de monitoramento: para o monitoramento, sugere-se avaliação contínua e sistemática, com planejamento de realização de hemogramas semestrais, para todos os trabalhadores expostos nesses locais, e quinzenais para os que tiverem alterações, constituindo uma série histórica de resultados dos exames para fins de vigilância e definição de conduta clínica. Caso a avaliação inicial seja indicativa de doença de medula óssea ou nas situações em que persistam as alterações nesse tempo mínimo de 45 dias, realizar biópsia de medula óssea e mielograma.

\section{Fluxograma}

Esses critérios e procedimento do manejo clínico segundo conduta e classificação foram apresentados no formato de fluxograma, cuja estrutura representa um desdobramento dos fluxos preconizados no protocolo de atenção à saúde dos trabalhadores expostos ao benzeno, do Ministério da Saúde ${ }^{14}$ (Figura 1).

Conforme a classificação, os indivíduos sob os Grupos 3 e 4, bem como os casos diagnosticados de benzenismo, deverão ser tratados como evento sentinela, indicando a necessidade de encaminhamento aos órgãos competentes para realização de ações de vigilância. Outros procedimentos operacionais, de acordo com a Portaria 776/2004 ${ }^{31}$, incluem o afastamento do trabalhador com benzenismo da fonte de exposição, com posterior encaminhamento à Previdência Social, emissão da CAT (Comunicação de Acidente de Trabalho) e notificação ao SINAN (Sistema de Informação de Agravos de Notificação).

\section{Discussão e recomendações}

Uma vez que não existe tratamento medicamentoso específico para os casos de intoxicação por vapores de combustíveis, especialmente por benzeno, o acompanhamento médico para os casos confirmados de intoxicação deve ser regular e de longo prazo. As intercorrências clínicas devem ser tratadas com precocidade e as perturbações de ordem psíquicas e sociais causadas aos indivíduos devem merecer atenção especializada em programas de saúde integrados sob o enfoque do trabalho. Considerando essas questões, essa proposta de classificação dos grupos permite instrumentalizar os profissionais de saúde no sentido de identificar e intervir na população trabalhadora exposta. O desenho apresentado em grupos possibilita o estabelecimento de fluxos de atendimento, definição de prioridades no atendimento, organização da rede de apoio ao diagnóstico e alimentação do sistema de Vigilância em Saúde do Trabalhador.

Importante destacar que os trabalhadores classificados no Grupo 1, apesar de não apresentarem alterações clínico-laboratoriais, devem constituir o grupo de expostos ao benzeno e serem acompanhados para adoção de medidas de proteção à saúde. Sendo assim, foi proposto um monitoramento por hemograma em períodos mínimos de seis meses ${ }^{32}$, podendo, no entanto, diante de alguma alteração clínica, ocorrer a diminuição de tal intervalo e a transferência do indivíduo para outros grupos hierárquicos.

Aqueles trabalhadores classificados sob o Grupo 2 são assim definidos pela impossibilidade de uma conclusão acerca da avaliação clínico-laboratorial. Dessa forma, é necessária uma avaliação mais precisa para a correta aplicação dos critérios de manejo, permitindo uma diferenciação entre os indivíduos classificados entre o Grupo 1 em relação aos demais grupos. A não realização da avaliação clínica impede a detecção de sinais e sintomas de uma possível intoxicação, como cefaleia, tonturas, alterações de memória, zumbido, mialgia, fraqueza, sonolência e infecções de repetição. A inexistência de um hemograma faz com que a avaliação perca seu parâmetro principal baseado nas alterações hematológicas como leucopenia (sendo a neutropenia a mais importante delas), outras citopenias, macrocitose persistente, eosinofilia, pontilhado em basófilo, hiposegmentação dos neutrófilos, linfocitopenia, plaquetopenia e macroplaquetas. Alguns desses achados clínico-laboratoriais podem, no entanto, ser inespecíficos, podendo desaparecer mesmo sem o afastamento do trabalhador.

Os próximos dois grupos requerem um monitoramento mais contínuo e sistemático ao longo do tempo de exposição, com programação de rotina de exames e investigações complementares e, em alguns casos, necessitam, ainda, de encaminhamento para outras especialidades, da mesma forma a estabelecer diagnóstico diferencial e avaliação da presença de comorbidades. A diferença básica entre os Grupos 3 e 4 é que, no primeiro, há a suspeição de existência de comorbidades e a definição do caso não é conclusiva e, diante de tal situação, a investigação do ambiente de trabalho é imprescindível, bem como o monitoramento clínico com realização de novos exames ou pareceres especializados mais frequentes. Alguns casos classificados sob o Grupo 3, tais como taxa de leucócitos superior ou igual a $4.500 / \mathrm{mm}^{3}$, 


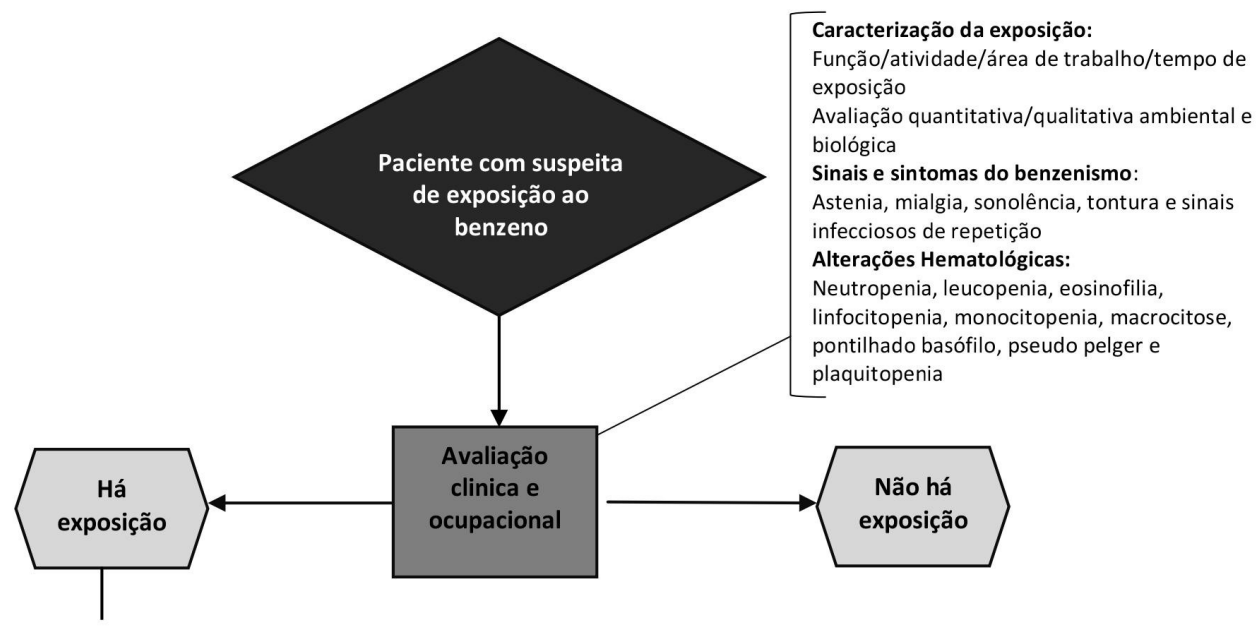

\section{CLASSIFICAÇ̃̃O DOS GRUPOS DE EXPOSTOS}

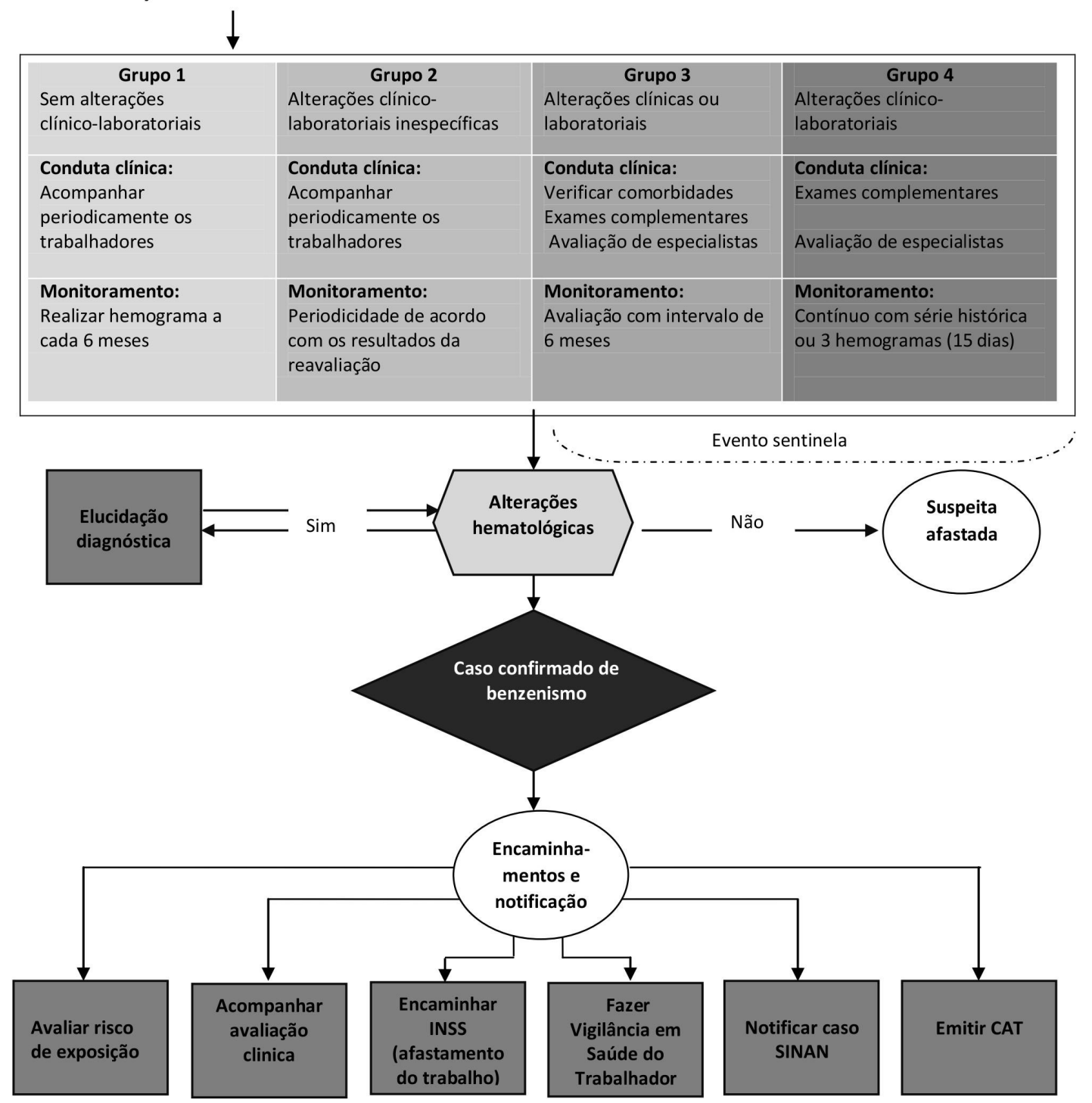

Fonte: Fluxograma elaborado pelos autores, seguindo os desdobramentos dos fluxos preconizados no protocolo de atenção à saúde dos trabalhadores expostos ao benzeno, do Ministério da Saúde ${ }^{\mathbf{1 4}}$

Notas: SINAN = Sistema de Informação de Agravo de Notificação, CAT = Comunicação de Acidente de Trabalho

Figura 1 Fluxograma do manejo clínico de trabalhadores expostos ao benzeno segundo conduta e classificação 
com neutropenia relativa persistente ou casos de anemia persistente, poderão ser enquadrados como Grupo 4. As situações em que ocorra a taxa global de leucócitos acima de 4.500 células $/ \mathrm{mm}^{3}$, mas com taxa global de neutrófilos menor ou igual a 1.000 células $/ \mathrm{mm}^{3}$, repetidas em dois exames, deverão ser enquadradas no Grupo 4.

Os indivíduos classificados no Grupo 4 são considerados casos, cabendo os procedimentos imediatos previstos no protocolo e anteriormente citados. Esse grupo de trabalhadores necessita de ações mais complexas e intersetoriais, pois o afastamento do risco da exposição deverá ser definitivo, considerando que o retorno à fonte de exposição agravará o quadro. Esse agravamento poderá levar ao surgimento de neoplasias do sistema hematopoiético.

A caracterização da exposição dos trabalhadores é realizada pela avaliação qualitativa do ambiente de trabalho. Entretanto, os testes quantitativos de indicadores ambientais e biológicos, como o ácido trans,trans-mucônico, ácido $S$-fenilmercaptúrico, ácido hipúrico e metil hipúrico, têm sua importância na determinação de níveis e limites de exposição em processos de avaliação de risco, em conjunto com a identificação de alterações bioquímicas precoces e outros efeitos subclínicos, tais como danos genotóxicos, estresse oxidativo, danos renais e hepáticos, cabendo salientar que sejam avaliados de forma coletiva e não individual. Reitera-se o uso desses indicadores como parte importante das ações em Vigilância em Saúde do Trabalhador.

Apesar deste estudo e outros tratarem da exposição aos derivados de petróleo, em especial à gasolina, como uma exposição ao benzeno (por sua relevância toxicológica e ocupacional), é de conhecimento a complexa composição dessas misturas. Sendo assim, é importante considerar os efeitos da exposição aos vapores de gasolina de forma geral, mesmo conhecendo os efeitos deletérios de cada composto sobre a saúde humana.

A principal vantagem desse processo de classificação clinico-laboratorial é seu uso para rastreamento ampliado em população de trabalhadores em qualquer nível de complexidade da rede de atenção à saúde, vinculando a saúde do trabalhador à atenção básica, dado o uso de procedimentos de fácil aplicação. Apesar disso, uma das limitações dessa classificação é a possibilidade de não identificar sinais clínicos em expostos, o que pode resultar em falta de acompanhamento mais regular em indivíduos com potencial de evolução para leucemia. Outra limitação é a dificuldade de atingir de forma ampliada os grupos de risco, uma vez que esse instrumento está direcionado aos serviços de saúde, que ainda contam com uma baixa cobertura no atendimento à população. Além disso, os trabalhadores tendem a procurar os serviços de saúde quando ocorrem manifestações de sinais clínicos de maior gravidade que, de acordo com a proposta de manejo clínico, já poderiam estar classificados como Grupos 3 ou 4.

Uma questão importante refere-se ao dilema da decisão do médico em relação ao retorno do trabalhador com um diagnóstico de benzenismo. Do ponto de vista clínico e ocupacional, a normalização ou estabilidade dos valores hematimétricos do sangue periférico de um trabalhador afastado pode levar um tempo médio de cinco anos ou mais. Entretanto, essa normalidade representa uma compensação fisiológica e não uma regeneração das células progenitoras e precursoras do sistema hematopoiético ${ }^{16}$. Portanto, não desconstitui a intoxicação e nem constitui critério para retorno ao ambiente ou à função com risco de exposição ao benzeno ou outros $\mathrm{COVs}^{33}$, pois uma vez afetada a medula óssea, a lesão é permanente. Dessa forma, se não houver comprovação de alterações tecnológicas suficientes para o controle das emissões de vapores de benzeno e outros COVs e a alocação para uma função sem exposição num PRC, do ponto de vista da saúde, a Previdência Social tem que readaptar esse trabalhador em outra função ou aposentá-lo.

\section{Conclusões}

Este processo de classificação dos achados clínico-laboratoriais dos trabalhadores expostos ao benzeno mostra-se potencialmente capaz de orientar os profissionais da RENAST para a conduta médica, no sentido de classificar as alterações hematológicas, sinais e sintomas relacionados com os potenciais riscos dessa exposição, portanto, realizar o monitoramento de forma sistemática e contínua, a partir do estabelecimento de rotinas e fluxos assistenciais definidos para os coletivos de trabalhadores. Importante salientar que se compreende essa classificação como uma parte integrada diretamente às ações de Vigilância em Saúde do Trabalhador, para a compreensão da experiência de exposição vivenciada pelos trabalhadores, as fontes de riscos e seus efeitos sobre a saúde.

Destaca-se que essa proposta de classificação não exclui a realização de outras ações de vigilância à saúde. Ela é parte integrada da atenção à saúde, em que os dados clínicos dos trabalhadores e os dados ambientais compõem os aspectos de biomonitoramento citados por Arnold et al. ${ }^{34}$, pois a avaliação individual compreende a quantificação dos efeitos e a avaliação ambiental envolve a identificação das fontes de exposição, que juntas fornecem informações importantes para limitar a exposição.

Portanto, a aplicação dessa classificação propõe a sistematização de análises clínicas e laboratoriais 
que, em conjunto com a história ocupacional, permite a identificação dos grupos expostos e, dentre eles, amplia a capacidade de análise dos casos pelas características comuns, bem como as possíveis intervenções, o que não seria possível caso fosse adotado apenas o critério individual.

O protocolo proposto é um elemento de conexão entre o acompanhamento clínico sistemático de atenção individual e a abordagem de saúde coletiva da vigilância em saúde, dando nexo ao conceito de atenção integral com ênfase na Vigilância em Saúde do Trabalhador preconizado na Política Nacional de Saúde do Trabalhador e da Trabalhadora (PNSTT) ${ }^{35}$. Além disso, é um importante instrumento para comparação com outros grupos de pesquisa e autoridades do campo da Saúde do Trabalhador e do âmbito das exposições ocupacionais ao benzeno.

\section{Contribuições de autoria}

Fonseca ASA, Costa DF, Dapper V, Valente D, Costa-Amaral IC, Menezes MAC, Larentis AL, Côrrea MJM, Mattos RCOC contribuíram substancialmente no projeto e delineamento, no levantamento de dados ou na sua análise e interpretação, elaboração do manuscrito, ou tiveram contribuição importante na sua revisão crítica e aprovação final da versão publicada. Machado JMH, Carvalho LVB, Alves SR e Sarcinelli PN contribuíram na elaboração do manuscrito ou tiveram contribuição importante na sua revisão crítica e aprovação final da versão publicada.

\section{Referências}

1. Keenan JJ, Gaffney SH, Galbraith DA, Beatty P, Paustenbach DJ. Gasoline: a complex chemical mixture, or a dangerous vehicle for benzene exposure? Chem Biol Interact. 2010;184(1-2):2935.

2. Agency for Toxic Substances and Disease Registry. Toxicological profile for benzene [Internet]. Atlanta: ATSDR; 2007. p. 438. Available from: https:/www.atsdr.cdc.gov/ toxprofiles/tp3.pdf

3. International Agency for Research on Cancer. Monographs on the evaluation of carcinogenic risks to humans. A review of human carcinogens: chemical agents and related occupations, soot, as found in occupational exposures of chimneysweeps. Vol. 100F. Lyon: IARC; 2012. 599 p.

4. Moura-Correa MJ, Jacobina AJR, Santos SA, Pinheiro RDC, Menezes MAC, Tavares AM, et al. Exposição ao benzeno em postos de revenda de combustíveis no Brasil: Rede de Vigilância em Saúde do Trabalhador (VISAT). Ciênc Saúde Coletiva. 2014;19(12):4637-48.

5. Correa SM, Arbilla G, Marques MRC, Oliveira KMPG. The impact of BTEX emissions from gas stations into the atmosphere. Atmospheric Pollut Res. 2012;3(2):163-9.

6. Costa, MFB. Estudo da aplicabilidade do ácido trans,trans-mucônico urinário como indicador biológico de exposição ao benzeno [tese]. [Rio de Janeiro]: Escola Nacional de Saúde Pública; 2001. Disponível em: http://teses.icict.fiocruz.br/pdf/ costamfbd.pdf

7. Coutrim MX, Carvalho LRF de, Arcuri ASA. Avaliação dos métodos analíticos para a determinação de metabólitos do benzeno como potenciais biomarcadores de exposição humana ao benzeno no ar. Quím Nova. 2000;23(5):653-63.
8. Machado JMH, Costa DF, Cardoso LM, Arcuri A. Alternativas e processos de vigilância em saúde do trabalhador relacionados à exposição ao benzeno no Brasil. Ciênc Saúde Coletiva. 2003;8(4):913-21.

9. Gouveia JLN, Nardocci AC. Acidentes em postos e sistemas retalhistas de combustíveis: subsídios para a vigilância em saúde ambiental. Eng Sanit Ambient. 2007;12(3):317-24.

10. International Programme on Chemical Safety. Benzene. World Heath Organization, Geneva. 2010. Disponível em: http://www.who.int/ipcs/ assessment/public_health/benzene/en/

11. Smith MT. advances in understanding benzene health effects and susceptibility. Annu Rev Public Health. 2010;31(1):133-48.

12. Zhu J, Wang H, Yang S, Guo L, Li Z, Wang W, et al. Comparison of toxicity of benzene metabolite hydroquinone in hematopoietic stem cells derived from murine embryonic yolk sac and adult bone marrow. PLoS ONE. 2013;8(8):e71153.

13. Snyder R. Leukemia and benzene. Int J Environ Res Public Health. 201214;9(12):2875-93.

14. Brasil. Ministério da Saúde. Secretaria de Atenção à Saúde. Departamento de Ações Programáticas Estratégicas. Risco químico: atenção à saúde dos trabalhadores expostos ao benzeno. Brasília: Ministério da Saúde; 2006. Disponível em: http:// bvsms.saude.gov.br/bvs/publicacoes/risco_saude_ trabalhadores_expostos_benzeno.pdf

15. Mendes R. Subsídios para a identificação de grupos ocupacionais de mais elevado risco de exposição. Bol Soc Bras Hematol Hemoter. 1987;20(144):135-47.

16. Augusto LG S, Novaes TCP. Ação médico-social no caso do benzenismo em Cubatão, São Paulo: uma 
abordagem interdisciplinar. Cad Saúde Pública. 1999;15(4):729-38.

17. Baxter PJ. Gases. In: Baxter PJ, Tar-Ching A, Cockcroft A, Durrington P, Harrington M, editors. Hunter's diseases of occupations. 10th ed. London: Hodder Arnold; 2010. p. 253-268.

18. Costa DF. Prevenção da exposição ao benzeno no Brasil [tese]. [São Paulo]: Universidade de São Paulo; 2009. Disponível em: http://www.teses.usp. br/teses/disponiveis/5/5144/tde-25092009-135349/ pt-br.php

19. Vlaanderen J, Lan Q, Kromhout H, Rothman N, Vermeulen R. Occupational benzene exposure and the risk of lymphoma subtypes: a meta-analysis of cohort studies incorporating three study quality dimensions. Environ Health Perspect. 2010;119(2):159-67.

20. Schnatter AR, Glass DC, Tang G, Irons RD, Rushton L. Myelodysplastic syndrome and benzene exposure among petroleum workers: an international pooled analysis. J Natl Cancer Inst. 2012;104(22):1724-37.

21. Linet MS, Yin S-N, Gilbert ES, Dores GM, Hayes RB, Vermeulen R, et al. A retrospective cohort study of cause-specific mortality and incidence of hematopoietic malignancies in Chinese benzene-exposed workers: Cancer in Chinese benzene workers. Int J Cancer. 2015;137(9):2184-97.

22. Ruiz MA, Vassallo J, Souza CA. Alterações hematológicas em pacientes expostos cronicamente ao benzeno. Rev Saúde Pública. 1993;27(2):145-51.

23. Ruiz MA, Lorand-Metze I, Augusto LGS, Souza CA. Characteristics of the hemopoietic abnormalities after chronic exposure to benzene. Leuk Res. 1994;18:29.

24. Levy BS, editor. Occupational and environmental health: recognizing and preventing disease and injury. 6th ed. New York: Oxford University Press; 2011. p. 854.

25. Tochetto TM, Quevedo, LS, Siqueira, MA. Condição auditiva de frentistas. Rev CEFAC. 2013;15(5):1137-47.

26. Unlu I, Kesici G, Basturk A, Kos M, Yılmaz O. A comparison of the effects of solvent and noise exposure on hearing, together and separately. Noise Health. 2014;16(73):410-5.
27. Wiwanitkit V. Classification of risk occupation for benzene exposure by urine trans, trans-munconic acid level. Asian Pac J Cancer Prev. 2006;7(1):149-50.

28. Fundacentro. Indicador biológico da exposição ocupacional ao benzeno. In: Seminário para revisão do protocolo para a utilização de indicador biológico da exposição ocupacional ao benzeno. São Paulo: Fundacentro; 2014.

29. Bogadi-Sare A, Zavalić M, Turk R. Utility of a routine medical surveillance program with benzene exposed workers. Am J Ind Med. 2003;44(5):467-73.

30. Brasil. Ministério da Saúde. Secretaria de Vigilância em Saúde. Diretoria Técnica de Gestão. Dengue: diagnóstico e manejo clínico: adulto e criança. 4ª ed. Brasília: Ministério da Saúde; 2013.

31. Brasil. Ministério da Saúde. Portaria $n^{0} 776$, de 28 de abril de 2004. Dispõe sobre a regulamentação dos procedimentos relativos à vigilância da saúde dos trabalhadores expostos ao benzeno, e dá outras providências. Diário Oficial da União, Brasília, DF, 29 abr 2004. Disponível em: http://www.saude. mt.gov.br/cosat/arquivo/1838/sobre-benzeno

32. Brasil. Ministério do Trabalho e Previdência Social. Portaria MTPS n ${ }^{\circ} 1109$, de 21 de setembro de 2016. Aprova o Anexo 2, Exposição Ocupacional ao Benzeno em Postos Revendedores de Combustíveis (PRC), da Norma Regulamentadora $\mathrm{n}^{\circ}$ 9, Programa de Prevenção de Riscos Ambientais (PPRA). Diário Oficial da União 22 set 2016.

33. Arcuri ASA, Costa DF, Possebon J. Efeitos da exposição ao benzeno para a saúde. São Paulo: Fundacentro; 2012. Disponível em: http://www. fundacentro.gov.br/biblioteca/biblioteca-digital/ publicacao/detalhe/2013/2/efeitos-da-exposicao-aobenzeno-para-a-saude-serie-benzeno-fasciculo-1

34. Arnold SM, Angerer J, Boogaard PJ, Hughes MF, O'Lone RB, Robison SH, et al. The use of biomonitoring data in exposure and human health risk assessment: benzene case study. Crit Rev Toxicol. 2013;43(2):119-53.

35. Brasil. Ministério da Saúde. Portaria $n^{0} 1.823$, de 23 de agosto de 2012. Institui a Política Nacional de Saúde do Trabalhador e da Trabalhadora. Diário Oficial da União, Brasília, DF, 24 ago 2012. Disponível em: http://bvsms.saude.gov.br/ bvs/saudelegis/gm/2012/prt1823_23_08_2012. html 